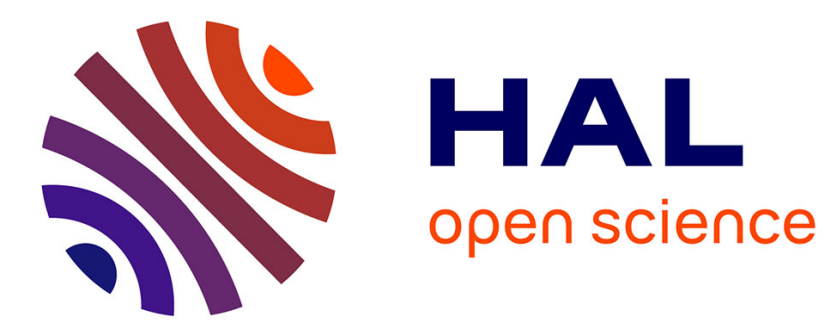

\title{
Wetting against the nap - how asperity inclination determines unidirectional spreading
}

Elise Contraires, Jérémie Teisseire, Elin Søndergård, Etienne Barthel

\section{To cite this version:}

Elise Contraires, Jérémie Teisseire, Elin Søndergård, Etienne Barthel. Wetting against the nap how asperity inclination determines unidirectional spreading. Soft Matter, 2016, 12, pp.6067-6072. 10.1039/C6SM00523C . hal-01336885

\section{HAL Id: hal-01336885 \\ https://hal.sorbonne-universite.fr/hal-01336885}

Submitted on 24 Jun 2016

HAL is a multi-disciplinary open access archive for the deposit and dissemination of scientific research documents, whether they are published or not. The documents may come from teaching and research institutions in France or abroad, or from public or private research centers.
L'archive ouverte pluridisciplinaire HAL, est destinée au dépôt et à la diffusion de documents scientifiques de niveau recherche, publiés ou non, émanant des établissements d'enseignement et de recherche français ou étrangers, des laboratoires publics ou privés. 


\title{
Journal Name
}

\section{ARTICLE TYPE}

\section{Wetting against the nap - how asperity inclination de- termines unidirectional spreading}

\author{
Elise Contraires, ${ }^{a}$ Jérémie Teisseire ${ }^{b, c}$ Elin Søndergård ${ }^{b}$ and Etienne Barthel ${ }^{d, e}$
}

\begin{abstract}
We have carried out wetting experiments on textured surfaces with high aspect ratio asperities in the Wenzel state. When inclination is imparted to the asperities, we observe a strictly unidirectional spreading opposite to the direction in which the asperities point. The advancing contact angle decreases markedly as inclination increases. A crude numerical analysis successfully accounts for this behaviour, highlighting the interplay between Gibbs pinning at the top of the structures and imbibition along the valleys between them. In Gibbs pinning non-linearities play a major role and we find that simple line averaging - i.e. a rule of mixture - cannot account for this evolution except for weak surface perturbations, i.e. large inclinations.
\end{abstract}

\section{Introduction}

With the advent of versatile microfabrication techniques, a nearly infinite variety of textured surfaces can be generated, with often surprising wetting properties ${ }^{1-10}$. Among them, anisotropic patterns sometimes induce unidirectional wetting, i.e. the triple line moves in one preferential direction. Directional wetting may result from simple geometrical asymmetry of basically planar motives ${ }^{11}$. However, a more ambitious route opens up in the third dimension: inclination imparted to high aspect ratio structures will also result in directionality. Of course, in this case, surface elaboration is much more complex. In practice, one possibility is to resort to biomorphological features ${ }^{12,13}$ or naturally occurring asymmetries during material elaboration ${ }^{14}$. A more controlled way is offered by more or less complex lithographic processes ${ }^{15,16}$, usually involving some oblique incidence modification step ${ }^{17-19}$.

Despite this large body of experimental evidence, the physical mechanisms of wetting in the presence of inclined structures are not completely clear. In fact, quite generally, it is found that anisotropic wetting proceeds in the direction of inclination of the

${ }^{a}$ Laboratoire de Tribologie et Dynamique des Systèmes, UMR5513, CNRS, Ecole Centrale de Lyon, 36 avenue Guy de Collongue, FR-69134 Ecully cedex, France

${ }^{b}$ Surface du Verre et Interfaces, UMR 125 CNRS/Saint-Gobain Recherche, 39 quai Lucien Lefranc, 93303 Aubervilliers, France

'PCRS, Saint-Gobain Recherche, 39 quai Lucien Lefranc 93303 Aubervilliers, France

${ }^{d}$ École Supérieure de Physique et de Chimie Industrielles de la Ville de Paris (ESPCI) ParisTech, PSL Research University, Sciences et Ingénierie de la matière Molle, CNRS UMR 7615, 10, Rue Vauquelin, F-75231 Paris Cedex 05, France

eSorbonne-Universités, UPMC Univ. Paris 06, SIMM, 10, Rue Vauquelin, F-75231 Paris Cedex 05, France

$\dagger$ Electronic Supplementary Information (ESI) available: videos of spreading droplet on textured GaSb surfaces with inclination angles of $0^{\circ}$ (00deg.avi) and $45^{\circ}$ (45deg.avi). See DOI: 10.1039/b000000x/ surface features. This observation can be explained by a purely 2D argument where the condition for the liquid to the reach next row is evaluated geometrically. This approach has proved quite effective to delineate the wetting phase diagram as a function of inclination angle ${ }^{17}$. Precise numerical investigations however demonstrate that the result holds only for relatively hydrophilic surfaces ${ }^{20}$. Experimentally, it was also found to fail for denser structures ${ }^{16}$.

In this context, a different modeling strategy has also been frequently used, which departs from the purely 2D picture. Heterogeneity in the lateral direction is taken into account to some extent by averaging some local contact condition along the triple line, resulting in an explicit evaluation of the macroscopic contact angle. This strategy has been regularly used to analyze wetting on heterogeneous surfaces, and the averaging has been carried out either on the contact angle itself ${ }^{21}$ or on its cosine ${ }^{22}$ (i.e. interfacial energy). For inclined surface features, the average will typically involve the local surface angle, based on Gibbs pinning condition. This strategy has been applied to dense polymer bushes ${ }^{14}$ and was found to account for the contact angle hysteresis adequately. Unfortunately, when used for gold films similarly deposited under oblique incidence, the same approach fails ${ }^{23}$. Another observation adds to the puzzle: while wetting in the direction of inclination is usually observed, the reverse can also occur. With partially hydrophobic half-cone shells ${ }^{19}$, it was found that the wetting direction is reversed, a result which the authors qualitatively ascribe to Gibbs pinning. Reverse wetting direction was also found for some high aspect ratio hybrid gold/polymer rods, where the inclination angle could be adjusted ${ }^{24}$. In this case, the anomalous wetting direction was ascribed to the chemical anisotropy of the Janus rods.

The picture which emerges is that for a good wetting and/or 
lower aspect ratios, simpler methods like 2D criteria or line averaging provide a reasonable picture of the macroscopic response of the liquid. However, when the surface is partially hydrophobic, and/or the asperities have high aspect ratios, these methods become increasingly inaccurate. In fact, for regular vertical textures, the issue has already been identified and handled by full numerical simulations ${ }^{25,26}$. The impact of geometry on wetting has been systematically studied by Semprebon et al. ${ }^{27}$ : they drew "phase" diagrams in which intrinsic wetting properties, asperity aspect ratio and density play major roles. Despite (or maybe because of) the very large number of wetting configurations they identified, depending on the geometrical details of the system under consideration, it is still not completely clear how surface patterns should be designed to purposefully tune wetting properties.

Therefore, varying the inclination of surface features is a good playground to test our understanding of the wetting of textured surfaces. Here we report on surface textures generated by diffuse ion beam etching. Small conical features are generated with a rather high density and high aspect ratio. Moreover the cone inclination can be adjusted easily, while the cone morphology is otherwise relatively unaffected. For such a structure, we find that wetting proceeds in the inverse direction. Measuring the advancing contact angle, we find a pronounced decrease as inclination angle increases. This variation can be accounted for by a relatively light 3D numerical simulation based on a simplified rendering of the pattern geometry. Our results demonstrate how pattern inclination drives a transition from an imbibition dominated regime for relatively flat surfaces to a Gibbs pinning dominated regime when the surface features stand out more markedly.

\section{Experimental}

\section{Surface preparation}

For surface patterning, (100) GaSb wafers were cut in $1 \mathrm{~cm}^{2}$ squares and exposed to diffuse $\mathrm{Ar}^{+}$sputtering (energy $\mathrm{U}=500 \mathrm{~V}$, flux $=0.2 \mathrm{~mA} / \mathrm{cm}^{2}$ ) for 10 minutes without heating. This treatment was applied in an ultrahigh vacuum chamber at a pressure of $10^{-7}$ mbar. After etching, the samples were kept in plastic boxes to protect them from dust contamination; they were typically used within a few days after fabrication. A full description of the technique can be found elsewhere ${ }^{28}$. As a result of ion bombardment the surfaces are covered with submicrometric cones (Fig. 1 left) with a narrow distribution of morphological parameters (width, height and intercone distance). Under these fabrication conditions, the cones are spatially disordered. Usually, abrasion is carried out at normal incidence. However when the sample is tilted relative to the ion beam axis, etching proceeds in such a way that the cones are tilted by the same angle from the normal of the surface (Fig. 1 right). For the present work, five different inclinations have been chosen $\left(0^{\circ}, 15^{\circ}, 30^{\circ}, 45^{\circ}\right.$ and $\left.60^{\circ}\right)$.

Some attempts at chemically modifying the surface by silane grafting have been carried out. Our endeavours were mainly limited by the surface chemistry of GaSb which is essentially unknown, especially after ion etching. Reasonable success was obtained at grafting fluorosilanes by vapour deposition. For more hydrophilic silanes, we obtained poor reproducibility and durability as soon as the surfaces were exposed to water. Presumably silane chemistry is not optimum in this case and only the non wetting modified surface can resist contact with water. As a result, all the measurements have been performed on surfaces which have not been further modified after ion etching, except for the relatively uninteresting case of the hydrophobized surfaces briefly touched upon below.

\section{Surface characterization and contact angle measurements}

GaSb surface morphology was characterized by imaging with a Gemini Scanning Electronic Microscope (ZEISS, Germany) at $10 \mathrm{kV}$. Top views were made directly on samples after wetting experiments (Fig. 1 left). Some samples were also cleaved with a diamond point at room temperature to observe cross-sections (Fig. 1 right). In addition to the cone inclination angle $\alpha$, we have also measured the average maximum and minimum angles in which the cones are included ( $\beta$ and $\gamma$ - inset Fig. 1$)$. These angles are shown in Table 1 as a function of etching incidence angle, along with the cone heights. Note that for an inclination angle $\alpha=60^{\circ}$, the cones are much shorter (Table 1). This abrupt morphology change is probably due to a different shadowing mechanism during etching at such a large incidence angle ${ }^{28}$. For a rough characterization of surface properties, static water contact angles were measured with a PGX goniometer (Testing Machin Inc, USA), placing a water droplet of $4 \mu \mathrm{L}$ on the surface. The static contact angle measured on smooth GaSb surfaces before etching was $89 \pm 5^{\circ}$. Dynamic contact angles were measured with a DSA100 goniometer (Krüss, Germany). The initial drop volume $5 \mu \mathrm{L}$ was increased by $2,5 \pm 0,5 \mu \mathrm{L}$. On the etched surfaces the contact angles were monitored on side views with the camera aligned normal to the orientation of the inclined cones. Data were processed with ImageJ software. Repeat experiments showed good reproducibility.
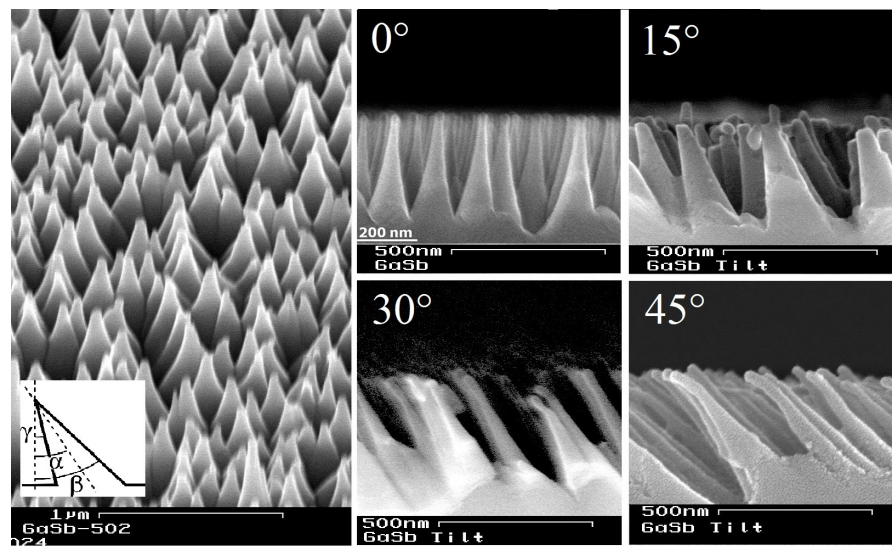

Fig. 1 SEM images of etched GaSb surfaces: left) oblique view for normal incidence etching; right) cross sections for normal incidence ( $0 \circ)$ and oblique incidence etching from 15 to $45^{\circ}$. Inset: geometrical description of a cone. 
Table 1 Structure morphology

\begin{tabular}{cccc}
\hline Fabr. tilt $\left(^{\circ}\right)$ & Meas. incl. $\left(^{\circ}\right)$ & In. angle $\left(^{\circ}\right)$ & Out. angle $\left(^{\circ}\right)$ \\
Cone height $(\mathrm{nm})$ & $\alpha_{\exp }$ & $\gamma$ & $\beta$ \\
$\mathrm{h}$ & $0 \pm$ & $-15 \pm 4$ & $15 \pm 4$ \\
\hline 0 & $17 \pm 2$ & $+6 \pm 2$ & $26 \pm 3$ \\
$403 \pm 160$ & $28 \pm 3$ & & \\
15 & & $+22 \pm 3$ & $35 \pm 3$ \\
$334 \pm 36$ & $43 \pm 3$ & $+36 \pm 4$ & $46 \pm 2$ \\
30 & & & \\
$446 \pm 32$ & $64 \pm 3$ & $+51 \pm 3$ & $70 \pm 2$ \\
45 & & & \\
$382 \pm 70$ & & & \\
60 & & & \\
$82 \pm 19$ & & & \\
\hline
\end{tabular}

\section{Results}

When a water droplet is deposited on a surface which has been hydrophobized after ion etching, a standard superhydrophobic Cassie state is found, with low hysteresis and no observable impact of cone inclination. In contrast, the as-etched GaSb surface is much more interesting. In this case water fills the valleys between the cones, i.e. the drop is in a Wenzel state. We observe that no receding contact angle can be measured: when the droplet is deflated, the triple line stays perfectly pinned. This is due to the very small feature size. We therefore focused on the advancing triple
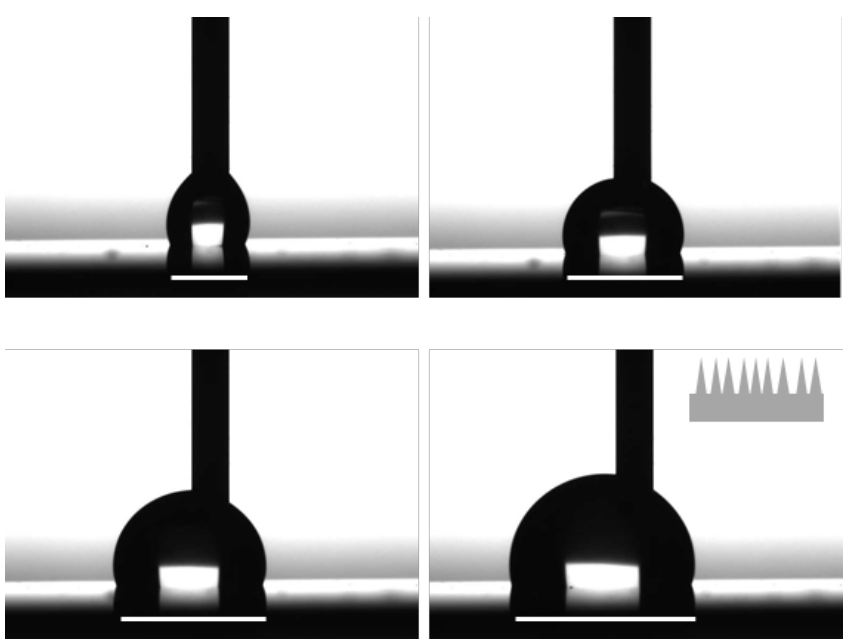

Fig. 2 Swelling of the droplet on patterned surfaces with vertical asperities $\left(\alpha=0^{\circ}\right)$. The white line under the drop shows the baseline of the droplet.

line. When the cones are vertical $\left(\alpha=0^{\circ}\right)$ we observe an isotropic motion of the triple line. After a phase of symmetric growth, the drop often starts to grow asymmetrically due to some degree of surface heterogeneity incurred during surface preparation but no preferential direction or orientation is found (Fig. 2 - see the video in the supplemental material as well). The results are very different when inclination is imparted to the textures $\left(\alpha>0^{\circ}\right)$. As an example, consider the swelling of a droplet on a surface with $\alpha=45^{\circ}$, shown in Fig. 3 (see the video in the supplemental material as well). Just after deposition, the drop spreads strictly unidirectionally: the front is pinned on one side and spreads on the other side. The needle is therefore always found on one side of the droplet. To connect with cone morphology, we call "with the cones" the direction in which the cones point, and "against the cones" the opposite direction. Interestingly, we find that the allowed line motion is not with the cones, as intuition suggests, but against the cones. This result also contrasts with most previous studies with tilted surface features ${ }^{14-17,20}$ where easy motion is found along the feature direction. Motion against the features has only been observed on sharp tilted cones, a system very similar to ours ${ }^{19}$ and asymmetric high aspect ratio polymeric posts ${ }^{24}$.

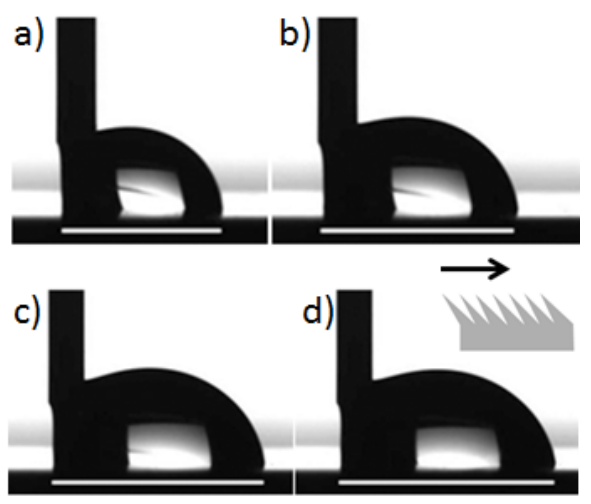

Fig. 3 Swelling of droplet on patterned surfaces $\left(\alpha=45^{\circ}\right)$. For clarity a white line has been added under the drop duplicating the base line of the droplet. The front is pinned in one side and moves against the direction in which the cones point.

From the side views we extracted the macroscopic advancing contact angle. Fig. 4 shows the evolution of this advancing contact angle as a function of inclination angle. In reference to the idea of Gibbs pinning, the data is plotted against maximum inclination angle $\beta$ (table 1 ) and not inclination angle $\alpha$. The difference is not very significant. We find that the advancing contact angle decreases strongly from $\theta_{a}=95^{\circ}$ for $\beta=15^{\circ}\left(\alpha=0^{\circ}\right)$ to $\theta_{a}$ $=77^{\circ}$ for $\beta=46^{\circ}\left(\alpha=43^{\circ}\right)$. The trend is not followed for $\beta=70^{\circ}$ as expected from the very different morphology of the surface features (Table 1). Therefore, this data point has not been included in the following analysis.

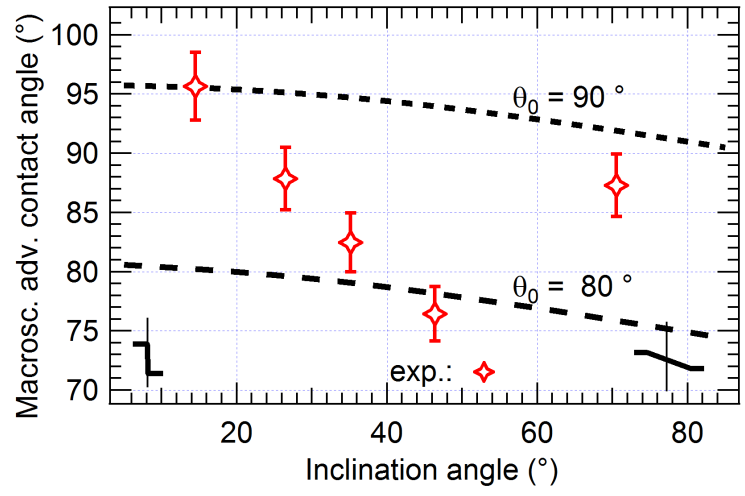

Fig. 4 Macroscopic advancing contact angle as a function of inclination angle: experimental data (red diamond) and average model (dashed line). 
We first compare these experimental results to a simple line average model ${ }^{16,19,26}$. Following the rule of mixture, we average wetting conditions along the triple line ${ }^{21,22}$. With $l$ the distance between patterns and $d$ the pattern width, the linear density along the triple line is $\lambda=d /(d+l)$. At depinning the triple line obeys the Gibbs condition at the top of the asperity and the macroscopic advancing contact angle $\theta_{\text {macro }}$ is approximated by:

$$
(\ell+d) \cos \theta_{\text {macro }}=(\ell+h) \cos \theta_{0}+d \cos \left(\frac{\pi}{2}-\beta+\theta_{0}\right)
$$

where $\theta_{0}$ is the microscopic contact angle and $\beta$ the pattern slope. The first term on the right hand side contains not only pattern width $l$ but also pattern height $h$, reflecting the possibility of imbibition due to interaction with the sides of the patterns. The second term is the contribution of the contact line on the edge of the tilted facet where it is assumed that the triple line depins when the liquid surface reaches the microscopic contact angle on the facet, as per Gibbs. Comparing typical results of this model to our data (Fig. $4-$ dashed lines, with $\theta_{0}=80^{\circ}$ or $90^{\circ}$ ), there clearly appears that the rule of mixture cannot reproduce the observed variation of the contact angle with inclination. Beyond simple surface energy averaging, there is a need for a more accurate description of the local distortions of the drop edge.

To investigate how a liquid wets surfaces with inclined textures in more detail, we have performed simulations using the minimization algorithm Surface Evolver ${ }^{29}$. With this algorithm, we generate minimal surfaces with appropriate, user defined boundary conditions. This is a versatile and numerically exact method to explore the equilibrium configurations of a portion of a full 3D drop/bubble surface in interaction with surfaces. The method is also numerically lightweight: for example, the interface energy terms are implemented through Stokes theorem, a very efficient scheme which however limits application to relatively simple geometries such as flat surfaces, cylinders and spheres. Therefore, to investigate the impact of our rather complicated on wetting, we have approximated the geometry of the inclined cones by a parallelepipedic post with an inclined front facet with angle $\beta$ (Fig. 5 a). Since we model the equilibrium configurations of a liquid surface, we also need to specify the liquid/solid boundary conditions. In the rather arbitrary unequilibrated initial configuration, the lower boundary of the liquid surface runs along the top and the edges of the post and also along the bottom surface. Periodic boundary conditions apply to the sides of the liquid surface so that what we model is effectively interaction with a row of posts. The upper boundary of the liquid surface touches a fictitious horizontal surface (a "ceiling") with an inner angle $\theta_{w}{ }^{30}$. The macroscopic contact angle $\theta_{\text {macro }}$ is set through $\theta_{\text {macro }}=\pi-\theta_{w}$. Equilibrating the surface numerically, we observe that it bulges out, and that the triple line overruns the top corners of the post to locally wet the front facet (Fig. 5 b). However, it remains arrested on the middle of the top edge of the facet, where the local contact angle with the facet plane is still smaller than $\theta_{0}$. Increasing $\theta_{\text {macro }}$ by small steps and equilibrating numerically at each step, we observe that the deformation of the liquid surface increases (Fig. 5 b) until, for a given angle, the triple line depins. At depinning, the distortion of the line is such that the microscopic contact angle is everywhere equal to $\theta_{0}$ on the tilted front facet of the pattern: the triple line can now move down this plane and wet the whole surface (Fig. 5 c). This threshold defines the macroscopic advancing contact angle.
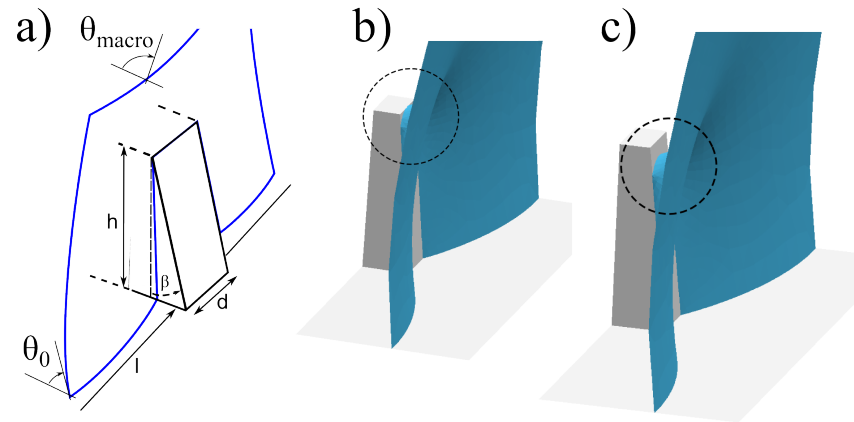

Fig. 5 a) Schematics of inclined surface feature modeled as an upright rectangular post with slanted facet (inclination angle $\beta$ ). The liquid surface is also shown in its initial, non equilibrium, geometry. The intrinsic contact angle between liquid and solid is $\theta_{0}$. A macroscopic contact angle $\theta_{\text {macro }}$ is imposed at the top of the liquid surface. During the simulation the macroscopic contact angle is increased stepwise. b) equilibrium configuration of the liquid surface for a macroscopic contact angle just below the depinning threshold. The liquid surface is strongly deformed but pinned in the middle of the top edge of the pattern. c) increasing the macroscopic contact angle again, the liquid surface depins and is now free to move down the facet. Depinning defines the macroscopic advancing contact angle.

Macroscopic advancing contact angles obtained by simulation are plotted as a function of inclination angle $\beta$ in Fig. 6 for different linear densities along the triple line $\lambda$ and compared to the results of the line average model (Eq. 1). Here the aspect ratio for the patterns is $h / d=3$ and for simplicity, the microscopic contact angle $\theta_{0}=90^{\circ}$ to suppress any effect of imbibition. There is a strong contrast between numerical simulations and rule of mixture (dashed lines in Fig. 6), especially for small inclination angles (i.e. for almost upright features). When inclination angle is close to $90^{\circ}$ on the other hand, numerical simulations and line averages give similar results, which means that when the surface corrugation is small, the rule of mixture provides a good first approximation. Indeed, when $\beta$ approaches $0^{\circ}$ on the other hand, the surface distortions involved in Gibbs pinning play a major role, resulting in a steep rise of the contact angle which is almost completely absent from the energy average approximation. The effect is seen more markedly when increasing the line density.

Investigating the impact of the microscopic contact angle $\theta_{0}$ at constant line density $\lambda=1 / 10$ (Fig. 7) we find that the macroscopic advancing contact angle decreases with decreasing $\theta_{0}$. The effect appears more markedly for large inclination angle. This evolution results from imbibition of the liquid in the valleys between the cones as the surface becomes more and more intrinsically wetting. Once again, the rule of mixture matches the simulations at large asperity inclinations, i.e. for low surface corrugation and Gibbs pinning plays an increasingly strong role as inclination angle decreases.

We have also plotted our experimental points on this graph. 


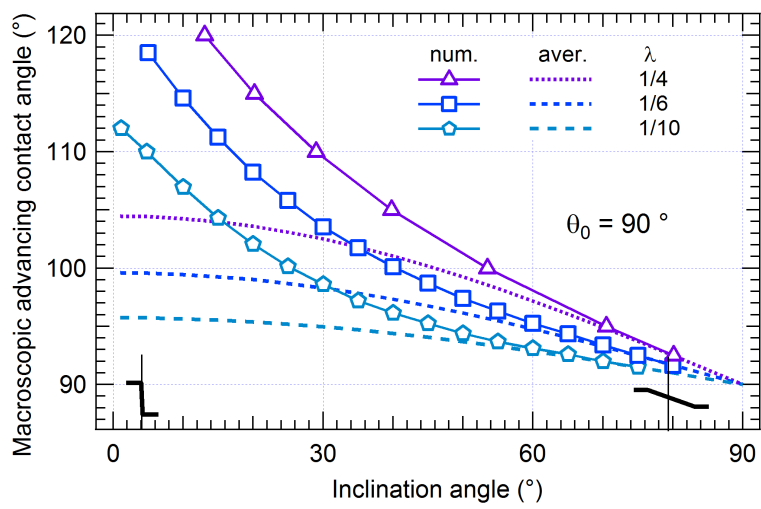

Fig. 6 Numerical macroscopic advancing contact angle plotted as a function of inclination angle. A microscopic contact angle $\theta_{0}=90^{\circ}$ and an aspect ratio of patterns $\mathrm{h} / \mathrm{d}=3$ are used for simulation. Three curves are obtained for different linear density of pattern along the triple line $(\lambda)$. The average model (dashed line) is plotted for comparison.

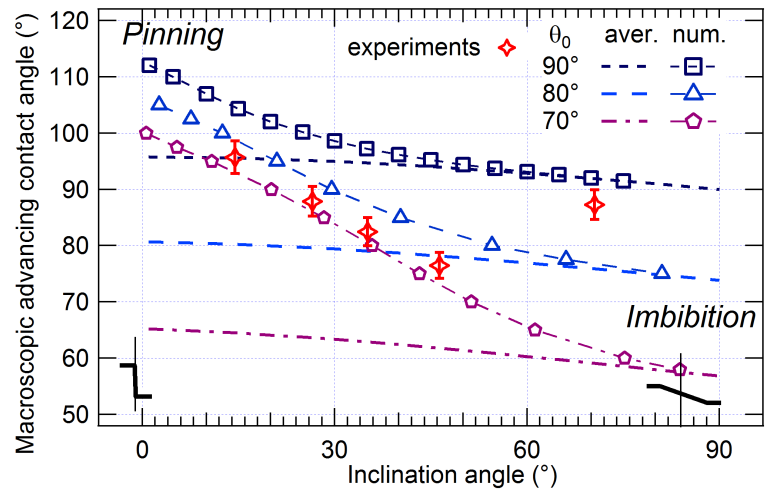

Fig. 7 Numerical simulations of the advancing contact angle plotted as a function of inclination angle for three different values of the microscopic contact angle $\theta_{0}=70^{\circ}, 80^{\circ}$ and $90^{\circ}$ for an aspect ratio $\mathrm{h} / \mathrm{d}$ $=3$ and a line density $\lambda=1 / 10$. The line average model (dashed line) is plotted for comparison. Our experimental results are also shown.

The evolution with inclination is reasonably accounted for by the numerical model using a microscopic contact angle $\theta_{0}$ close to $70^{\circ}$. Again the surface with $\alpha=60^{\circ}$ does not comply with this scheme, due to the very different morphology registered for this angle value.

\section{Discussion}

Our surfaces are covered with small cones of high aspect ratio, in quite large density. The inclination angle of these cones can be adjusted and we have investigated the impact of inclination on wetting. With non-zero inclination, asymmetric wetting is found. Along the cone direction, the triple line is pinned: it can move only against the cones. In comparison, this anisotropic movement of the triple line is not observed on symmetric structures, with vertical cones $\left(\alpha=0^{\circ}\right)$. Moreover we find a strong variation of the macroscopic advancing contact angle as a function of inclination (Fig. 4). A simple line averaging model (Eq. 1) produces only a moot dependence with inclination which is unable to account for this evolution (Fig. 7). By numerical simulations, with a very simplified geometry, we have found that local pinning of the triple line at the top of the pillars plays in fact a dominant role for low inclinations, i.e. when the features stand close to upright and this model reproduces the steep evolution of the contact angle found in the experiments.

In the simulation, for an estimated line density $\lambda=1 / 10$, a good match is found for a microscopic contact angle $\theta_{0}=70^{\circ}$. This is of course an effective value, due to the crude description of the geometry. More precise simulations would involve modeling the exact cone shape and both geometrical and spatial distributions, which is beyond the scope of this paper. This effective value of the microscopic contact angle is somewhat lower than the value measured on the pristine GaSb surface, which probably results from a modification of the surface chemistry during the etching process, especially due to the gallium mobility ${ }^{28}$. The fact that this microscopic contact angle is lower than $90^{\circ}$ is consistent with the imbibition we observe, mediated by the additional wetting area provided by the side faces of the patterns (Wenzel factor). This contribution is especially apparent at high inclination angles (Fig. 7) where we can see that the macroscopic advancing contact angle indeed becomes smaller than the microscopic contact angle. Imbibition actually makes the contact angle evolution with inclination stronger: Gibbs pinning holds off wetting, especially for rather upright features, and imbibition promotes wetting, especially for rather shallow features. What we observe is therefore a gradual transition between a regime dominated by pinning for fairly vertical patterns to a regime dominated by imbibition for large inclination angles, i.e. a nearly flat surface. If the microscopic contact angle is adequately selected, the range of macroscopic advancing contact angles spanned when the textures tilt from vertical to almost horizontal will be large precisely because of this transition between two very contrasted mechanisms. The present numerical model, however crude in the description of the geometry, does include the two main effects at work in wetting: imbibition and Gibbs pinning. Thanks to the numerical scheme, and in contrast to the rule of mixture, the non-linearities inherent to Gibbs pinning with high aspect ratio asperities are modeled accurately.

Finally, our results also fully rationalize the directionality of the wetting process. The sharp rise of the macroscopic advancing contact angle as inclination increases makes it clear that for negative inclination, depinning is even more difficult or impossible. Negative inclination is indeed the configuration met by the triple line when it tries to move along the cones. This kind of geometrical configuration with an overhang is similar to the reentrant geometry which imparts enhanced stability to the Cassie state ${ }^{7}$. This is the reason why on our surfaces, wetting is asymmetric, along the direction with positive inclination, i.e. against the nap.

\section{Conclusions}

We have developed original surface patterns: small, dense cones with a well defined inclination angle and high aspect ratio. We have studied the impact of this inclination angle on wetting. Strikingly, wetting is completely unidirectional, and quite unexpectedly, the triple line moves opposite to the direction in which the cones point. Our simulations show that this directionality is 
due to pinning of the triple line at the top of the cones: an overhang is considerably more difficult to overcome than a sloping down facet. This pinning mechanism also accounts for a strong dependence of contact angle on inclination angle, much stronger than a simple line averaging model can predict. The amplitude of this transition is actually increased by imbibition. These results emphasize the role of pinning in the macroscopic response of patterned surfaces, a non-linear deformation process which cannot be encapsulated in a simple energy averaging procedure, except in the limited number of cases where these deformations are actually small, i.e. a nearly flat surface.

\section{References}

1 A. Paterson and M. Fermigier, Phys. Fluids, 1997, 9, 2210.

2 J. Bico, U. Thiele and D. Quéré, Colloids Surf., A, 2002, 206, 41-46.

3 S. Moulinet, C. Guthmann and E. Rolley, Eur. Phys. J. E, 2002, 8, 437-443.

4 G. McHale, S. Aquil, N. Shirtcliffe, M. Newton and H. Erbil, Langmuir, 2005, 21, 11053-11060.

5 C. Dorrer and J. Rühe, Langmuir, 2006, 22, 7652-7657.

6 L. Gao and T. J. McCarthy, Langmuir, 2007, 23, 3762-3765.

7 A. Tuteja, W. Choi, J. Mabry, G. McKinley and R. Cohen, Proc. Nat. Acad. Sci., 2008, 105, 18200.

8 P. Brunet, Soft Matter, 2012, 8, 11294-11301.

9 P. Papadopoulos, X. Deng, L. Mammen, D.-M. Drotlef, G. Battagliarin, C. Li, K. Muî́lllen, K. Landfester, A. del Campo, H.-J. Butt et al., Langmuir, 2012, 28, 8392-8398.

10 K. Davitt, M. S. Pettersen and E. Rolley, Langmuir, 2013, 29, 6884-6894.

11 V. Jokinen, M. Leinikka and S. Franssila, Adv. Mater., 2009, 21, 4835-4838.

12 Y. Zheng, X. Gao and L. Jiang, Soft Matter, 2007, 3, 178-182.

13 M. Prakash and J. W. Bush, Int. J. Non Linear Mech., 2011, 46, 607-615.

14 N. A. Malvadkar, M. J. Hancock, K. Sekeroglu, W. J. Dressick and M. C. Demirel, Nat. Mater., 2010, 9, 1023-1028.

15 M. K. Kwak, H.-E. Jeong, T.-i. Kim, H. Yoon and K. Y. Suh, Soft Matter, 2010, 6, 1849-1857.

16 C. Q. Lai and W. Choi, Adv. Mater. Interfaces, 2015, 2, year.

17 K.-H. Chu, R. Xiao and E. N. Wang, Nat. Mater., 2010, 9, 413417.

18 T.-i. Kim and K. Y. Suh, Soft Matter, 2009, 5, 4131-4135.

19 B. Ai, L. Wang, H. Möhwald, Y. Yu, Z. Zhao, Z. Zhou, G. Zhang and Q. Lin, Sci. Rep., 2014, 4, year.

20 A. Cavalli, M. L. Blow and J. M. Yeomans, Soft Matter, 2013, 9, 6862-6866.

21 C. Extrand, Langmuir, 2002, 18, 7991-7999.

22 W. Choi, A.Tuteja, J. Mabry, R. Cohen and G. McKinley, J. Colloid Interf. Sci., 2009, 339, 208-216.

23 L. Kubus, H. Erdogan, E. Piskin and G. Demirel, Soft Matter, 2012, 8, 11704-11707.

24 C.-M. Chen, C.-L. Chiang and S. Yang, Langmuir, 2015, 31, 9523-9526.
25 C. Dorrer and J. Rühe, Langmuir, 2008, 24, 1959-1964.

26 P. S. Forsberg, C. Priest, M. Brinkmann, R. Sedev and J. Ralston, Langmuir, 2010, 26, 860-865.

27 C. Semprebon, S. Herminghaus and M. Brinkmann, Soft Matter, 2012, 8, 6301-6309.

28 S. Le Roy, E. Barthel, N. Brun, A. Lelarge and E. Søndergård, J. Appl. Phys., 2009, 106, 094308.

29 K. Brakke, Exp. Math., 1992, 1, 141-165.

30 B. M. Mognetti and J. M. Yeomans, Langmuir, 2010, 26, 18162-18168. 